\title{
Clinical Profile of Patients Admitted in Hyperglycaemic Emergencies and Their Outcomes in Patients at a Tertiary Care Hospital in Southern India - a Retrospective Study
}

\author{
Dheeraj Jain ${ }^{1}$, Sivaraman $A^{1}$, Balasubramaniam² \\ ${ }^{1}$ Assistant Professor, Indira Gandhi Medical College \& Research Institute, Puducherry, ${ }^{2}$ Associate Professor, Indira Gandhi Medical College \& Research Institute, \\ Puducherry.
}

\section{Abstract}

Background: To study the clinical profile of patients with diabetic ketoacidosis (DKA) and hyperosmolar hyperglycemic state (HHS) and their outcome in a tertiary care hospital in Puducherry. Subjects and Methods: This was a retrospective study of patients with DKA and HHS admitted in Indira Gandhi Medical College \& Research Institute (IGMCRI), Puducherry over a period of two years. Data on precipitating factors, clinical features, serum electrolytes, duration of hospital stay and mortality were obtained. Results: 100 patients were included in the study. 84 patients had DKA and 16 patients had HHS. Of the 84 patients with DKA 65 (77.4\%) were diagnosed as Type 1 DM and 19 patients as Type 2 DM (22.4\%). Commonest precipitating factor for DKA was missed treatment (61\%) followed by Infection. Total number of deaths in DKA was 9. Out of 19 type 2 patients with DKA,5 expired which was much higher to those to with type 1 DM with DKA (4 out of 65). The commonest cause of death was infectious (sepsis related) cause, with UTI being the commonest (40\%). In HHS patients the mean age of presentation was 68.3. HHS was associated with higher mortality compared to DKA. Coma, altered sensorium (6 out of 16 ), seizures (4 out of 16) was seen in significant number of patients with HHS. BU, creatinine, osmolality was significantly elevated in patients with HHS who expired compared to survivors. Most common cause of death in HHS was sepsis (infection) related with UTI (40\%). Conclusion: DKA is common in patients with T2DM in our setup. Over 50\% of the patients presenting with DKA or HHS have no previous diagnosis of DM. Infections and noncompliance are important precipitants of hyperglycaemic emergencies.

Keywords: Diabetic Ketoacidosis, Hyperglycaemic Hyperosmolar State, precipitating factors.

Corresponding Author: Dr.A. Sivaraman, Assistant professor, Dept of general medicine, Indira Gandhi medical college and research institute Puducherry-1.

Received: August 2019

Accepted: August 2019

\section{Introduction}

Hyperosmolar hyperglycaemic state (HHS) and Diabetic Ketoacidosis (DKA) represent two distinct metabolic derangements manifested by insulin deficiency and severe hyperglycaemia. ${ }^{[1,2]}$ HHS occurs when there is insulin deficiency relative to insulin requirements causing hyperglycaemia, which in turn leads to dehydration, ultimately resulting in a severe hyperosmolar state. DKA occurs in the setting of more severe insulin deficiency, when low circulating levels of insulin lead not only to hyperglycaemia and dehydration but also to the production of ketone bodies and acidosis.

Diabetic ketoacidosis (DKA) is a frequent cause of morbidity and mortality in Type 1 Diabetes patients while HHS is common in type 2 diabetes mellitus (T2DM).

The risk for death with DKA has been about $4 \% 3$ but some studies report lower mortality rates in recent years. Deaths are concentrated primarily in the elderly.

Patient education and self-monitoring tools can prevent DKA. When it does occur, management that follows evidence-based treatment principles, which include hydration, insulin therapy, potassium repletion, and correction of the precipitating factor, can prevent DKArelated morbidity and mortality.

HHS is characterized by severe hyperglycemia, hyperosmolality, and dehydration in the absence of significant ketoacidosis. These metabolic derangements result from the combination of absolute or relative insulin deficiency and an increase in counter regulatory hormones (glucagon, catecholamines, Cortisol, and growth hormone). The present study is an aimed to determine the pattern of DKA at a teaching hospital, to assess the clinical and biochemical characteristics of the patients and the precipitating factors for hyperglycemic emergencies, and to correlate these parameters with the outcome.

\section{Definition of terms}

The criteria used to diagnose DKA in the patients included in the study were: typical clinical features such as hyperventilation, vomiting and dehydration; hyperglycemia $>250 \mathrm{mg} / \mathrm{dL}$; arterial blood $\mathrm{pH}<7.3$ and/or serum bicarbonate $<15 \mathrm{mmol} / \mathrm{L}$; and presence of ketonuria. 
HHS was defined by the presence of severe hyperglycaemia (glucose $>600 \mathrm{mg} / \mathrm{dL}$ ), and hyperosmolarity $>320 \mathrm{mOsmol} / \mathrm{L}$ with little or no ketonemia/ketonuria. ${ }^{[4-6]}$

The severity of DKA was defined according to the American Diabetes Association (ADA) criteria as mild (arterial blood $\mathrm{pH} 7.25-7.30$ ), moderate ( $\mathrm{pH} 7.00-7.24)$ or severe $(\mathrm{pH}<7.00) .6$ Effective osmolality was calculated using the formula: [( $2 \mathrm{x}$ sodium $)+$ plasma glucose/18], with normal values being $290 \mathrm{mmol} / \mathrm{kg}$ water.

\section{Subjects and Methods}

A descriptive retrospective analysis of records of patients admitted in IGMCRI and diagnosed with DKA/HHS over a period of 2 years was done. Data of patients who presented as Type 1 or Type 2 Diabetes Mellitus with diagnosis of Diabetic Ketoacidosis (DKA) or Hyperosmolar Hyperglycemic State (HHS) were included in the study. Patient records with incomplete case data were excluded from the study. During this period 277 suspected DKA/HHS cases were admitted to the medical ward and intensive care unit of the hospital. Out of these, only 100 patients were included in this study because the others did not meet the inclusion criteria of the study.

The following variables were recorded: age, sex, Duration of Diabetes, precipitating factors for DKA, duration of illness before seeking medical advice, clinical features, laboratory findings at presentation with DKA (plasma glucose, serum blood urea nitrogen, serum creatinine, serum sodium and potassium, urine ketones, arterial blood gas analysis, plasma bicarbonate and plasma osmolality), associated complications, duration of hospitalization and outcome. Patients were classified as having Type 1 or Type 2 Diabetes based on the age of diagnosis, if DKA was first presentation, lean or obese phenotype, family history and on the basis of medical records.

The data were analysed using SPSS, version 11.0. Differences between groups were tested statistically using the chi-square test and independent samples $t$-test. Differences were considered statistically significant at Pvalue $<0.05$.

\section{Results}

Out of 100 patients studied. 84 patients had DKA and 16 patients had HHS.

\section{Diabetic Ketoacidosis}

Of the 84 patients with DKA, 65 (77.4\%) were diagnosed as Type $1 \mathrm{DM}$ and 19 patients as Type 2 DM (22.4\%). According to the ADA criteria, 13 of the episodes were mild, 43 were moderate and 28 were severe. There were 11 episodes in newly diagnosed cases and 73 episodes in previously known cases of DM.

Of the total 84 number of cases of DKA 46 were in males and 38 in females. [Table 1] The mean age of all patients was 29.88 years. Patients with Type 2 DM were significantly older than those with Type 1 DM (mean age
48.4 versus 24.6).

The commonest presenting symptoms in this study were general weakness and fatigue (96\%), osmotic symptoms $(68 \%)$, abdominal pain (56\%), fever in (52\%) nausea and/or vomiting (58\%).

The duration of symptoms was 3.18 days with Type $1 \mathrm{DM}$ and 3.11 days in Type 2 .There was no significant difference in duration of symptoms or duration of DM.

Missed treatment (complete stoppage or reduction of insulin dose or frequency) was the commonest precipitating factor for the episode of DKA in $62 \%$ cases. Infection was the next most common reason in $38.1 \%$ cases.

Mean RBS at presentation in Type 1 was 461 and in Type 2DM was 483 with a range of 302-600. Both hypokalaemia and hyperkalaemia seen in patients with DKA (rangel.9-6). Average $\mathrm{HC} 03$ was 9.9. Urea, creatinine was significantly elevated in Type 2DM compared to Type 1 (p.OOl). HbAlC was significantly higher in Type 2 DM compared to Type 1 (p.003).

Table 1: Comparison of DKA and HHS based on sex of the patient

\begin{tabular}{|l|l|l|}
\hline & $\begin{array}{l}\text { Diabetic } \\
\text { ketoacidosis }\end{array}$ & $\begin{array}{l}\text { Hyperosmolar } \\
\text { hyperglycemic state }\end{array}$ \\
\hline Number of patients & 84 & 16 \\
\hline Sex & & \\
\hline Males & 46 & 9 \\
\hline Females & 38 & 7 \\
\hline
\end{tabular}

Table 2: Comparison of DKA and HHS

\begin{tabular}{|l|l|l|l|}
\hline & $\begin{array}{l}\text { Total cases } \\
\text { DKA }\end{array}$ & Type 2 DKA & HHS \\
\cline { 2 - 4 } & Mean & Mean & Mean \\
\hline Number & 84 & 19 & 16 \\
\hline Age & 29.8 & 48.42 & 68.93 \\
\hline Duration Of Illness & 3.16 & 3.105 & 3 \\
\hline Duration Of Dm & 4.05 & 4.26 & 4.25 \\
\hline SBP & 111.96 & 107.526 & 111.8 \\
\hline DBP & 68.41 & 66.89 & 68.8 \\
\hline PR & 85.27 & 81.947 & 82 \\
\hline RR & 28.5 & 30.105 & 31.3 \\
\hline BMI & 22.32 & 23.81 & 25.87 \\
\hline Vomiting & 49 & 10 & 4 \\
\hline Abdominal Pain & 52 & 11 & 2 \\
\hline Seizures & 0 & 0 & 4 \\
\hline RBS & 466.23 & 483 & 532 \\
\hline NA & 132.97 & 132.47 & 141 \\
\hline K & 4.34 & 4.07 & 4.06 \\
\hline BU & 38.77 & 60 & 77.56 \\
\hline CREAT & 1.21 & 1.63 & 1.9 \\
\hline HC03 & 9.8 & 9.42 & 23.1 \\
\hline PH & 7.10 & 7.15 & 7.40 \\
\hline IVF & 5.82 & 4.736 & 4.68 \\
\hline IV Insulin Dose & 46.1 & 46 & 45.6 \\
\hline Mortality NO & 9 & $5(19)$ & $5(16)$ \\
\hline Osmolality & 301.6 & 303.2 & 38.4 \\
\hline & & & \\
\hline & & & \\
\hline
\end{tabular}

The mean hospital stay was 4.77 days. Total number of patients who died was 9. Out of 19 Type 2 patients with DKA, 5 expired which was much higher to those to with Type 1 DM and DKA (4 out of 65). The mortality rate was significantly higher among patients with Type 2 DM than those with Type $1(40.0 \%$ versus $6.6 \%)(\mathrm{P}<0.001)$ ODDS RATIO 5.44. Patients who died were significantly older 
(28.44 vs. 43). BP, RR, HC03, CREATININE and TOTAL WBC COUNTS were the variables which significantly correlated with death when compared with survivors. Commonest cause of death in DKA was infectious (sepsis related) cause, with UTI being the commonest. [Table $3 \&$ 4]

\section{Hyperosmolar hyperglycaemic state}

Mean age of HHS was 68.3. Duration of symptoms before presentation was significantly higher in patients who expired. Coma, altered sensorium (6 out of 16), seizures (8 out of 16) was seen in significant number of patients. BU, creatinine osmolality was significantly elevated in patients with HHS who expired compared to survivors. Commonest cause of death in HHS was sepsis (infection) related with UTI $(40 \%)$. HHS was associated with higher mortality compared to DKA. [Table 3 \& 4]

\section{Table 3: Mortality Data in Patients With DKA/HHS}

\begin{tabular}{|l|l|l|}
\hline Type & Number & Percentage \\
\hline TYPE 1 DKA & $4 / 65$ & 6.15 \\
\hline TYPE 2 DKA & $5 / 19$ & 26.31 \\
\hline HHS & $5 / 16$ & 31.25 \\
\hline
\end{tabular}

\begin{tabular}{|l|l|l|l|}
\hline Table 4: Cause of Death DKA and HHS \\
\hline CAUSE & TYPE 1 & TYPE 2 & HHS \\
\hline UTI & 2 & 2 & 2 \\
\hline PNEUMONIA & 1 & 1 & 1 \\
\hline $\begin{array}{l}\text { SEVERE ACIDOSIS } \\
\text { WITH SEPSIS }\end{array}$ & 1 & & 1 \\
\hline MI & 0 & 1 & 1 \\
\hline CVA & 0 & 1 & 0 \\
\hline
\end{tabular}

\section{Discussion}

Despite the small sample size of this study, and the fact that it was a retrospective analysis conducted at a teaching hospital, all of which are important limitations, this study gives a basic profile of DKA /HHS in this part of the world. While most of the episodes of DKA in our study were due to Type $1 \mathrm{DM}, 22.4 \%$ were due to Type $2 \mathrm{DM}$. Although DKA is usually considered as a unique marker for Type 1 DM these reports clearly demonstrate that it can occur among Type 2 diabetic patients. ${ }^{[7]}$ This fact is increasingly recognized throughout the world. Females were $44.8 \%$ of all DKA admissions.

The mean age of all patients (29.88 years). However, patients with Type $2 \mathrm{DM}$ were significantly older than those with Type 1 (42.22 and 28.4 years respectively) presumably because Type $2 \mathrm{DM}$ tends to develop in adults older than 40 years while Type 1 DM usually occurs at a much younger age.

\section{Clinical features at admission}

Poor drug compliance was the major precipitating factor of DKA in our patients (72.6\%). Infections were the second most common precipitating factor (38.1). In developed countries infection is the commonest precipitating factor of DKA, while insulin disruption ranks second, perhaps due to better patient education.

\section{Biochemical abnormalities}

The great majority of patients $(75 \%)$ were normokalaemic at presentation and the raised serum creatinine seen in most of the DKA patients can also be a false elevation, as acetoacetate artificially raises measured creatinine in the standard colorimetric assay.

\section{Outcome}

The mean hospital stay was 4.77 days. The mortality rate of our DKA cases was high $(10 \%)$. This high mortality rates may reflect variability in access to medical services delayed presentation as well as poor patient education.

DKA patients with Type 2 DM had a significantly higher mortality rate than patients with Type 1 DM, probably because Type 2 DM patients were significantly older and more likely to have co morbidity. Overall, patients who died were significantly older, with more co morbidity, had hypotension, tachycardia, higher serum creatinine, and lower blood $\mathrm{pH}$.

Patients with HHS were older compared to DKA which is a well known fact. Duration of symptoms before presentation was significantly higher in patients who expired. Coma, altered sensorium, seizures was seen in significant number of patients with HHS compared to DKA. BU, creatinine, osmolality was significantly elevated in patients with HHS who expired compared to survivors.

\section{Conclusion}

To conclude, our study showed that hyperglycaemic emergencies can be a common presentation previously undiagnosed diabetes and that DKA is common with T2DM in our setup. Non-compliance and infections are the common precipitants. There is a need to educate people about the symptoms of DM and its complications and to put DM screening programs in place for persons above the age of 30 years and for those with risk factors for DM for early detection and treatment of DM.

\section{References}

1. Alberti KGMM. Diabetic ketoacidosis - aspects of management. In: Ledingham JG, ed.Tenth advanced medicine symposium. Tunbridge Wells: Pitman Medical, 1974:68-72.

2. Kitabchi AE, UmpierrezGE, Miles JM, et al. Hyperglycemic crises in adult patients with Diabetes. Diabetes Care. 2009;32 1335-43

3. Efstathiou SP, Tsiakou AG, Tsioulos DI, et al. A mortality prediction model in diabetic ketoacidosis. Clin Endocrinol (Oxf) 2002;57:595-601.

4. American Diabetes Association. 15. Diabetes Care in the Hospital: Standards of Medical Care in Diabetes-2019. Diabetes Care. 2019 Jan 1;42(Supplement 1):S173-81.

5. Gosmanov AR, Gosmanova EO, Kitabchi AE. Hyperglycemic crises: diabetic ketoacidosis (DKA), and hyperglycemic hyperosmolar state (HHS). InEndotext [Internet] 2018 May 17. MDText. com, Inc.

6. Dhatariya KK, Vellanki P. Treatment of diabetic ketoacidosis (DKA)/hyperglycemic hyperosmolar state (HHS): novel advances in the management of hyperglycemic crises (UK versus USA). Current diabetes reports. 2017 May 1;17(5):33.

7. Misra S, Oliver NS, Dornhorst A. Diabetic ketoacidosis: not always due to type 1 diabetes. Bmj. 2013 Jun 10;346:f3501. 
Copyright: ( ) the author(s), 2019. It is an open-access article distributed under the terms of the Creative Commons Attribution License (CC BY 4.0), which permits authors to retain ownership of the copyright for their content, and allow anyone to download, reuse, reprint, modify, distribute and/or copy the content as long as the original authors and source are cited.

How to cite this article: Jain D, Sivaraman A, Balasubramaniam. Clinical Profile of Patients Admitted in Hyperglycaemic Emergencies and Their Outcomes in Patients at a Tertiary Care Hospital in Southern India - a Retrospective Study. Asian J. Med. Res. 2019;8(3):ME23-ME26. DOI: dx.doi.org/10.21276/ajmr.2019.8.3.ME8

Source of Support: Nil, Conflict of Interest: None declared. 